\title{
Thermal Dileptons from a Nonperturbative Quark-Gluon Phase
}

\author{
C.-H. Lee ${ }^{a}$, J. Wirstam $^{b}$, I. Zahed ${ }^{a}$ and T.H. Hansson ${ }^{b}$ \\ a) Department of Physics \& Astronomy, SUNY at Stony Brook, Stony Brook, NY 11794, USA \\ b) Institute of Theoretical Physics, University of Stockholm, \\ Box 6730, S-113 85 Stockholm, Sweden
}

\begin{abstract}
Assuming that gluon condensates are important even above the deconfining phase transition, we develop a model for the dilepton yield from a quark gluon plasma. Using a simple fire ball description of a heavy ion collision, and various estimates of the strengths of the gluon condensates, we compare our predicted dilepton yields with those observed in the CERES and HELIOS experiments at CERN. The simple model gives an adequate description of the data, and in particular it explains the observed considerable enhancement of the yield in the low mass region.
\end{abstract}

\section{INTRODUCTION AND SUMMARY}

Extensive lattice simulations have shown that QCD exhibits a phase transition at a temperature of the order of the infrared scale $\Lambda$. This transition is believed to be a confinement-deconfinement transition for heavy quarks, and a chiral transition for light quarks. The high temperature phase is characterized by a small coupling constant $g(T)$. Unfortunately, perturbation theory in $g(T)$ is plagued with infrared singularities since the magnetic sector is non-perturbative at all temperatures.

High temperature lattice [1, 2, and instanton simulations [3] seem to concur on the fact that in the vicinity of the critical temperature, $T_{c}$, the chromoelectric and chromomagnetic condensates are still substantial. A number of theoretical arguments, both perturbative [ [] and nonperturbative [5], suggests that in the Euclidean formulation, the high temperature phase of QCD exhibits strong $A_{4} A_{4}$ correlations. It has even been argued that the global color symmetry might be spontaneously broken with the appearance of an $A_{4}$ condensate [5.6]. Also, lattice simulations show that chiral symmetry is restored at the critical temperature, so that the quark condensate can be set to zero above $T_{c}$.

In the present analysis we will incorporate these three important observations that are pertinent to QCD near and above $T_{c}$; the quark condensate vanishes, the chromoelectric and chromomagnetic correlations remain non-zero, and strong correlations develop in the static part of the $A_{4}$ gauge field that can be described by a local $A_{4}^{2}$ condensate. In the spirit of the QCD sum rule method, we will not address the complicated dynamical questions of how the gluon condensates come about, but simply assume that they exist. We will also restrict our discussion to only those with dimension 2, $\left\langle\frac{\alpha_{s}}{\pi} A_{4}^{2}\right\rangle$, and $4,\left\langle\frac{\alpha_{s}}{\pi} E^{2}\right\rangle$ and $\left\langle\frac{\alpha_{s}}{\pi} B^{2}\right\rangle$, following an earlier suggestion by two of us [7]. The value of the condensates can only be estimated using non-perturbative calculations such as lattice QCD, and at high temperature, the scale is expected to be set by the mass gap in the magnetic sector which is $\sim g^{2} T$.

That chromoelectric and chromomagnetic condensates could be present in the high temperature phase is not very surprising, and their size can be roughly estimated using the bulk energy and momentum measurements at finite temperature on the lattice. The analysis in [2] gives the values : $\left\langle\frac{\alpha_{s}}{\pi} E^{2}\right\rangle \sim\left\langle\frac{\alpha_{s}}{\pi} B^{2}\right\rangle \sim(200 \mathrm{MeV})^{4}$

The presence of an $A_{4}^{2}$ condensate is more controversial [8], so we will give a summary of the arguments presented in [7] for why such a condensate should be considered. The basic observation is that the Wilson line (or Polyakov loop) operator $W=\operatorname{Tr} \operatorname{Pexp}\left(i \int_{0}^{\beta} d x_{4} A_{4}\left(x_{4}\right)\right)$, is a gauge invariant operator that is a good order parameter for the pure glue theory. Although this operator is non-local, it is possible to relate it to a string of local operators by choosing the static gauge $\partial_{4} A_{4}=0$. For example, in an $\mathrm{SU}(2)$ theory we have $\langle W\rangle=\left\langle 1+\cos \left(\frac{\beta}{2} A_{4}\right)\right\rangle$, and one could now try to use factorization to extract $\left\langle A_{4}^{2}\right\rangle$. Here we will simply equate the condensate to the magnetic mass, i.e. we will typically use, $\left\langle\frac{\alpha_{s}}{\pi} A_{4}^{2}\right\rangle / T^{2} \sim \frac{\alpha_{s}}{\pi}\left(g^{2}\right)^{2} \sim 16 \pi \alpha_{s}^{3}$, which is about 0.4 for $\alpha_{s} \sim 0.2$ This estimate is also supported by a lattice calculation in pure Yang-Mills [5]. Indeed, an analysis of the gluon-propagator in four-dimensions in Landau-gauge gives $a^{2}\left\langle\left(g A_{4}\right)^{2}\right\rangle \sim 1 / 3$ on a $4 \times 8^{3}$ lattice, which is equivalent to $\left\langle\frac{\alpha_{s}}{\pi} A_{4}^{2}\right\rangle / T^{2} \sim 0.4$. A direct reconstruction of this condensate from the dimensionally reduced theory has proven to be more subtle [ 8$]$.

In this letter, we present a calculation that suggests that the gluon condensates strongly affect the emission rates of low mass dileptons from the plasma. Our calculation will be very naive. We will simply assume that the quarkantiquark annihilation processes that give rise to the dileptons occur in the presence of low momentum fluctuating background gluon fields. By averaging over the background fields, we get leading corrections to the emission rates proportional to the various condensates. Note that in doing so we take the concept of a fluctuating background field at face value and perform the calculations directly in the time-like region assuming that the temperature is high enough for perturbation theory to be useful. Thus our method is very different from the QCD sum rules, where the background field method is just a convenient tool to calculate coefficient functions in the operator product expansion 
which is then used in the deep Euclidean region.1 However, since the emission rates can be expressed in terms of current-current correlation functions we can use the standard QCD sum rule techniques to calculate the condensate contributions. How this is done in detail, especially when it comes to gauge choice, is explained in [7]. To calculate the contribution from the $A_{4}^{2}$ condensate we will use the following observation: A constant potential $A_{4}$ is the same as an imaginary (color) chemical potential, $\mu^{a}$, and thus one can obtain the term $\sim\left\langle A_{4}^{2}\right\rangle$ by using a grand canonical ensemble for the quarks with a chemical potential $\mu^{a}=i A_{4}^{a}$, averaged over the arbitrary directions, $a$, in color space, and pick up the term $\sim \mu^{2}$. (We have also checked that this procedure gives the same result as the standard fixed point gauge methods used in [7].)

At this point we want to stress that although our approach is inspired by the above theoretical arguments, it is basically phenomenological, and should primarily be judged by how successfully it can accommodate data. The theoretical uncertainties are not limited to the assumptions we made about the non-perturbative corrections to correlations functions in a high temperature quark gluon plasma. We also have to make several assumptions about the physics of the heavy ion collision, and we will use an oversimplified model, namely that of an expanding fireball containing only the plasma phase. The main motivation for using such a simple model (the details are given in section III below) is to demonstrate that the gluon condensate effects are important. Hopefully more realistic models e.g. including regions with mixed or hadronic phases, would also exhibit this sensitivity to the condensates. Also in the transition regime, the pure and mixed quark condensates may still be relevant.

Having said all this, we will demonstrate that a naive fire ball model combined with a simple parametrization of the production amplitudes based on the above ideas, and with the condensate parameters at the values suggested by the scale arguments, is quite successful in explaining the dilepton data from the CERES and HELIOS relativistic heavy ion experiments. The rest of this paper, where this claim is substantiated, is organized as follows: In section II, we calculate the effects of the leading order condensates on the dilepton emission rates from a thermalized quark-gluon phase. In section III], we use a simple fire-ball model to estimate the dilepton yield, and compare to the CERES and HELIOS experiments. In section IV, we show that our emission rates are also consistent with the recent analysis of the CERES results using different $p_{t}$ cuts.

\section{EMISSION RATES}

In a translationally invariant, but non-perturbative, quark-gluon phase, the rate $\mathbf{R}$ of dileptons produced in a unit four volume is directly related to the electromagnetic current-current correlation function [10,11]. For a pair of leptons with mass $m_{l}$ and momenta $p_{1,2}$, the rate per unit invariant momentum $q=p_{1}+p_{2}$ is ,

$$
\frac{d \mathbf{R}}{d^{4} q}=-\frac{\alpha^{2}}{6 \pi^{3} q^{2}}\left(1+\frac{2 m_{l}^{2}}{q^{2}}\right)\left(1-\frac{4 m_{l}^{2}}{q^{2}}\right)^{1 / 2} \mathbf{W}(q),
$$

where $\alpha=e^{2} / 4 \pi$ is the fine structure constant, and

$$
\mathbf{W}(q)=\int d^{4} x e^{-i q \cdot x} \operatorname{Tr}\left(\mathbf{P} e^{i A_{4}^{a} \mathbf{Q}^{a}} e^{-(\mathbf{H}-\mathbf{F}) / T} \mathbf{J}^{\mu}(x) \mathbf{J}_{\mu}(0)\right)
$$

Here $e \mathbf{J}_{\mu}$ is the hadronic part of the electromagnetic current, $\mathbf{H}$ the hadronic Hamiltonian, $\mathbf{F}$ the free energy, $T$ the temperature, and $\mathbf{Q}^{a}$ a global color generator. (As mentioned above the direction, $a$, is arbitrary but fixed.) $\mathbf{W}(q)$ is related to the retarded current-current correlation function by,

$$
\mathbf{W}(q)=\frac{2}{e^{q^{0} / T}-1} \operatorname{Im} \mathbf{W}^{R}(q)
$$

where

$$
\mathbf{W}^{R}(q)=i \int d^{4} x e^{i q \cdot x} \operatorname{Tr}\left(\mathbf{P} e^{i A_{4}^{3} \mathbf{Q}^{3}} e^{-(\mathbf{H}-\mathbf{F}) / T} \mathbf{R}^{\star}\left(\mathbf{J}^{\mu}(x) \mathbf{J}_{\mu}(0)\right)\right)
$$

\footnotetext{
${ }^{1}$ A work similar in spirit to ours is the calculation by Patkos and Sakai 12 of the nonperturbative magnetic contribution to the $e^{+} e^{-}$annihilation rate. That calculation was inspired by the so called Copenhagen vacuum based on fluctuating magnetic fluxtubes.
} 
and $\mathbf{R}^{\star}$ is the retarded product. The leading contribution to the retarded function is condensate independent, and is just the "Born" $q \bar{q}$ annihilation term, which for massless quarks becomes,

$$
\operatorname{Im} \mathbf{W}^{R}=\frac{1}{4 \pi}\left(N_{c} \sum_{q=u, d, s} e_{q}^{2}\right) q^{2}\left[1+\frac{2 T}{|\vec{q}|} \ln \left(\frac{n_{+}}{n_{-}}\right)\right]
$$

where $N_{c}$ is the number of colors, $e_{q}$ the charge of the quarks, and $n_{ \pm}=1 /\left(e^{\left(q_{0} \pm|\vec{q}|\right) / 2 T}+1\right)$. At $T=0$ this is just the $R_{\geq 3}$ ratio well known from $e^{+} e^{-}$annihilation

$$
R_{\geq 3}\left(q^{2}\right)=\sum_{i} \frac{\sigma_{i}(\geq 3 \pi)}{\sigma\left(e^{+} e^{-} \rightarrow \mu^{+} \mu^{-}\right)}=-\left.\frac{4 \pi}{q^{2}} \operatorname{Im} \mathbf{W}_{0}^{R}\right|_{T=0}=N_{c} \sum_{q} e_{q}^{2}
$$

Using only the three light flavors, $R_{\geq 3}$ is 2 .

As mentioned above, the contribution of the $A_{4} A_{4}$ condensate to the retarded function $\mathbf{W}^{R}$ can easily be calculated by picking up the term quadratic in the imaginary colored chemical potential $A_{4}^{a}$. The result is

$$
\operatorname{Im} \mathbf{W}_{11}^{R}=\frac{1}{4 \pi}\left(N_{c} \sum_{q} e_{q}^{2}\right) q^{2}\left[\left\langle\frac{\alpha_{s}}{\pi} A_{4}^{2}\right\rangle\left(\frac{4 \pi^{2}}{T|\vec{q}|}\right)\left(n_{+}\left(1-n_{+}\right)-n_{-}\left(1-n_{-}\right)\right)\right] .
$$

According to the estimates in the previous section, $\beta \equiv\left\langle\frac{\alpha_{s}}{\pi} A_{4}^{2}\right\rangle / T^{2}$ is of the order of 0.4 . Note that ( 7 ) has the same sign as (5) and will thus increase the dilepton yield. In the next section, we will see that this effect can be rather dramatic in the invariant mass region studied in the CERES experiments.

The contribution of the chromoelectric and chromomagnetic condensates to the retarded function can be calculated using the methods in []], and the result for $q^{2}>0$ is,

$$
\operatorname{Im} \mathbf{W}_{12}^{R}=\frac{1}{4 \pi}\left(N_{c} \sum_{q} e_{q}^{2}\right)\left[\left(-\frac{1}{6}\left\langle\frac{\alpha_{s}}{\pi} E^{2}\right\rangle+\frac{1}{3}\left\langle\frac{\alpha_{s}}{\pi} B^{2}\right\rangle\right)\left(\frac{4 \pi^{2}}{T|\vec{q}|}\right)\left(n_{+}\left(1-n_{+}\right)-n_{-}\left(1-n_{-}\right)\right)\right]
$$

The temperature dependence of ( 8 is the same as that of $(7)$ and can be understood from the condition of detailed balance. Note that the emission rate is enhanced by a chromomagnetic field and depleted by a chromoelectric field as expected ${ }^{2}$, and also that all condensate contributions vanish at $T=0$.

\section{RESULTS}

In Fig. 11 we show the dilepton emission rates at temperature $T=150 \mathrm{MeV}$, using the parameters, $\delta \equiv$ $\left\langle\frac{\alpha_{s}}{\pi} E^{2}\right\rangle /(200 \mathrm{MeV})^{4}=\left\langle\frac{\alpha_{s}}{\pi} B^{2}\right\rangle /(200 \mathrm{MeV})^{4}[2]$. The contribution from the non-perturbative plasma, i.e. perturbative plus condensate contributions (dotted and dash-dotted lines) should be compared to the purely perturbative part (thick solid line) and, as a reference, to that of a gas of mesons (thin solid line) [13] . The condensate contribution to the rates are sizable in the $500 \mathrm{MeV}$ region, and the effect of the $\left\langle A_{4}^{2}\right\rangle$ condensate, $\beta$, dominates that of the chromoelectric and chromomagnetic ones, $\delta$, for masses above $250 \mathrm{MeV}$. The dependence on both condensates $\beta$ and $\delta$ is negligible at energies higher than $2 \mathrm{GeV}$, and above $2.5 \mathrm{GeV}$ the perturbative plasma gives the same result as that of a hadron gas, as expected from duality. In the Dalitz region $(<200 \mathrm{MeV})$ the effects of both the electric and magnetic condensates are appreciable and dominates the perturbative part by orders of magnitude. This observation maybe important for photon emission as well.

\footnotetext{
${ }^{2}$ Roughly speaking, the $e^{+} e^{-}$pairs are repelled from each other in the CM frame in the presence of a chromoelectric field, and deflected in the same direction in the presence of a chromomagnetic field.
} 


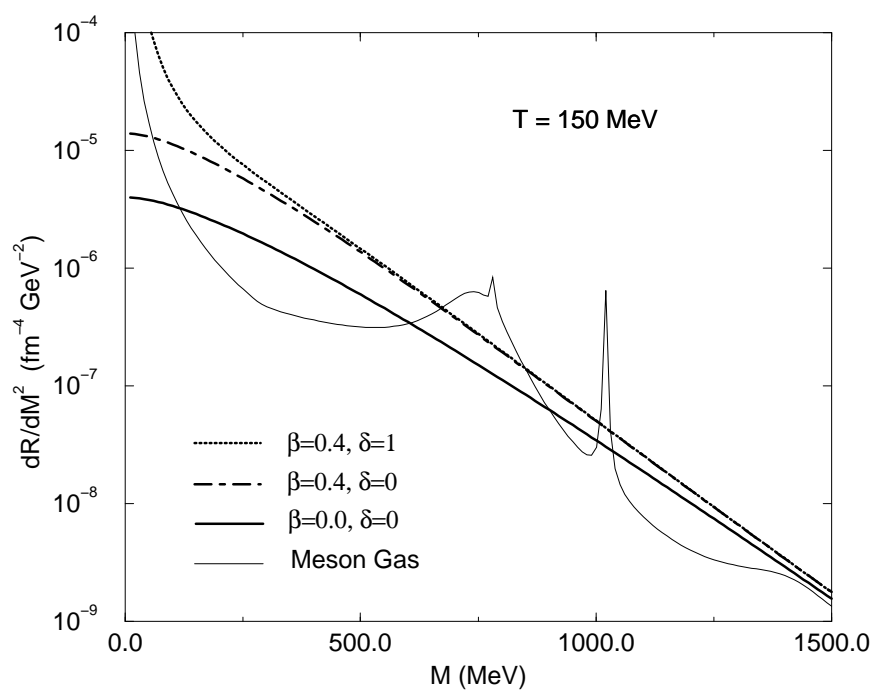

FIG. 1. Dielectron rates at $\mathrm{T}=150 \mathrm{MeV} . \beta \equiv\left\langle\frac{\alpha_{s}}{\pi} A_{4}^{2}\right\rangle / T^{2}$ and $\delta \equiv\left\langle\frac{\alpha_{s}}{\pi} E^{2}\right\rangle /(200 \mathrm{MeV})^{4}=\left\langle\frac{\alpha_{s}}{\pi} B^{2}\right\rangle /(200 \mathrm{MeV})^{4}$. See text.

In a heavy-ion collision the electromagnetic emission occurs from various stages of the collision process, and hence at various temperatures. In this part, we will focus on the emission rates in the low and intermediate mass region (up to $1.5 \mathrm{GeV}$ ). We will assume that the dileptons emanate from a simple fire ball composed of the nonperturbative plasma phase only. The fire ball expansion is assumed to be homogeneous, and we will use a standard parametrization of volume and temperature,

$$
\begin{aligned}
& V(t)=V_{0}\left(1+\frac{t}{t_{0}}\right)^{3} \\
& T(t)=\left(T_{i}-T_{\infty}\right) e^{-t / \tau}+T_{\infty} .
\end{aligned}
$$

The parameters in (9) regarding the initial and freeze-out temperature will be taken from present transport calculations [14,15. We will not try to justify this point except by saying that it allows us to define a space-time volume for the "hot" spot in the heavy-ion collision that is consistent with the one used for an interacting meson-phase 13 . 15 . Hence $t_{0}=10 \mathrm{fm} / \mathrm{c}, T_{i}=170 \mathrm{MeV}, T_{\infty}=110 \mathrm{MeV}, \tau=8 \mathrm{fm} / \mathrm{c}$, and the value of $V_{0}$ is absorbed into the over-all normalization constant $N_{0} V_{0}=6.76 \times 10^{-7} \mathrm{fm}^{3}$. The freeze-out time will be set to $t_{f . o .}=10 \mathrm{fm} / \mathrm{c}$.

Using (9) and the above rate, the final expression for the integrated emission rate per unit rapidity $\eta$ and invariant $\operatorname{mass} M$ is

$$
\frac{d N / d \eta d M}{d N_{c h} / d \eta}(M)=N_{0} M \int_{0}^{t_{f . o .}} d t V(t) \int \frac{d^{3} q}{q_{0}} A\left(q_{0}, q^{2}\right) \frac{d \mathbf{R}}{d^{4} q} .
$$

The acceptance function $A$ enforces the detector cut $p_{t}>200 \mathrm{MeV}, 2.1<\eta<2.65$, and $\Theta_{e e}>35$ mrad for CERES. In Fig. 2, we show the evolved emission rates for CERES. The enhancement is sizable and goes in the direction of the data in the $500 \mathrm{MeV}$ range in CERES. Again, the nonperturbative QCD results are indicated by the dotted line for the condensates we quoted, the dashed-dotted line (without the E- and B-condensates), while the perturbative QCD result is indicated by the solid line and the meson result by the thin solid line. 


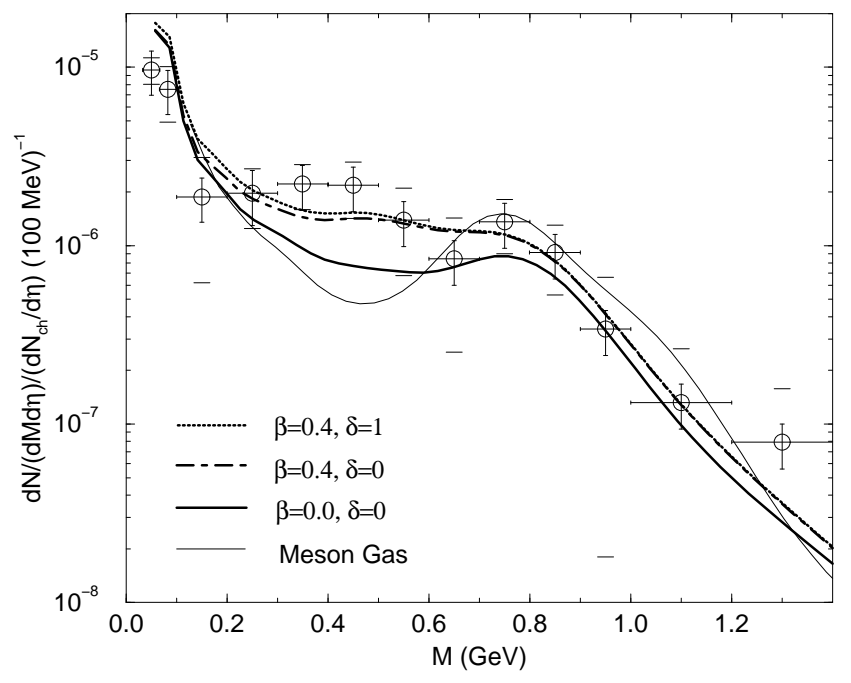

FIG. 2. Dielectron rates for CERES S-Au experiment. See text.

For HELIOS-3, (10) can be used by integrating over $\eta$ with the cut $m_{t} \geq 4(7-2 \eta), m_{t} \geq \sqrt{\left(2 m_{\mu}\right)^{2}+(15 / \cosh (\eta))^{2}}$. In Fig. 3, we show the evolved emission rates for HELIOS. For this energy range the effect of chromoelectric and chromomagnetic condensates are small irrespective of the specific value for the thermal condensates as indicated in Figs. 1 and 2. The enhancement is compatible with the HELIOS data, and slightly overshoots both the data and the meson gas in the $1 \mathrm{GeV}$ region.

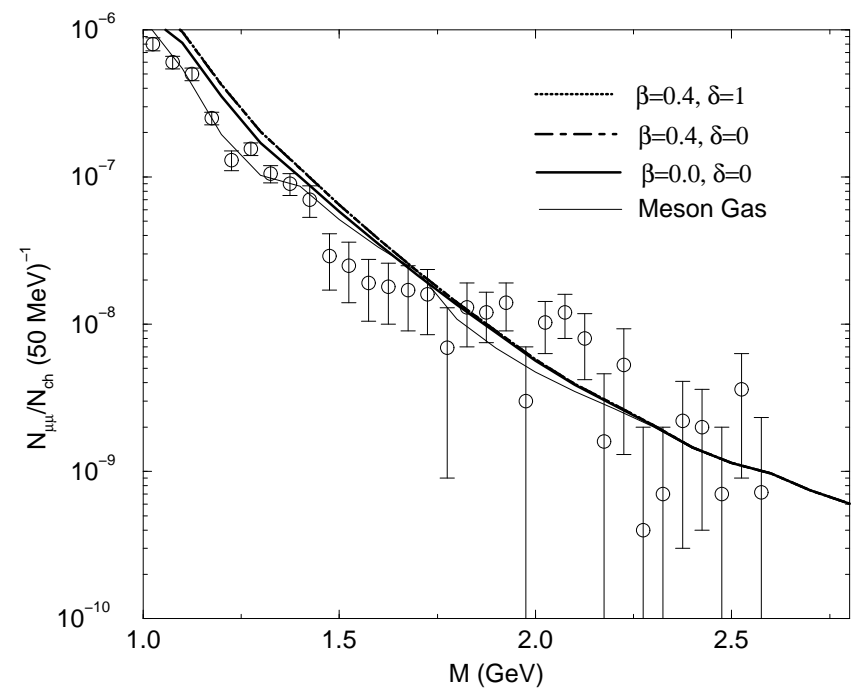

FIG. 3. Dimuon rates for HELIOS-3 S-W experiment. See text.

\section{IV. $P_{T}$ CUTS}

Another important check on the nonperturbative emission rates can be obtained by using the recent $p_{t}$ cut analysis by the CERES collaboration to probe the transverse momentum content of the dilepton emission rates in the fireball. Our results versus the data are shown in Figs. 16 , with and without the chromoelectric and chromomagnetic condensates. The $p_{t}$ dependent effects caused by the thermal condensates are most dramatic in the low $p_{t}$ region 
(Fig. (1), where there is a sizable condensate effect, mainly due to $\beta$. The condensate contributions are in the right direction around the low mass dielectron enhancement, and give important additions to the perturbative result.

In the high $p_{t}$ region, Fig. 0 , the resulting condensate effects are rather small, and the curve is consistent with the present data. Therefore, the total transverse momentum content (Fig. 6) is quite accurately reproduced by the addition of the gluon condensates.

In summary, we find it encouraging that not only the dilepton emission rates, but also the observed low mass-region $p_{t}$ enhancement is, at least partially, explained by our simple model, and we think that this further strengthens our proposal that nonperturbative condensate effects play a role in the plasma phase of heavy ion collisions.

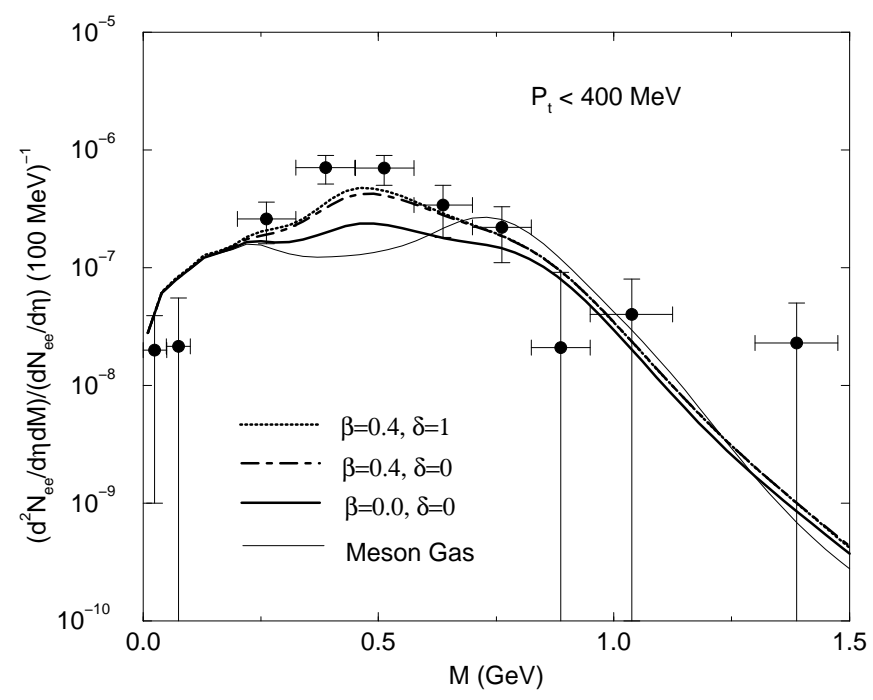

FIG. 4. Dielectron rates for low $p_{t}$ of CERES Pb-Au experiment.

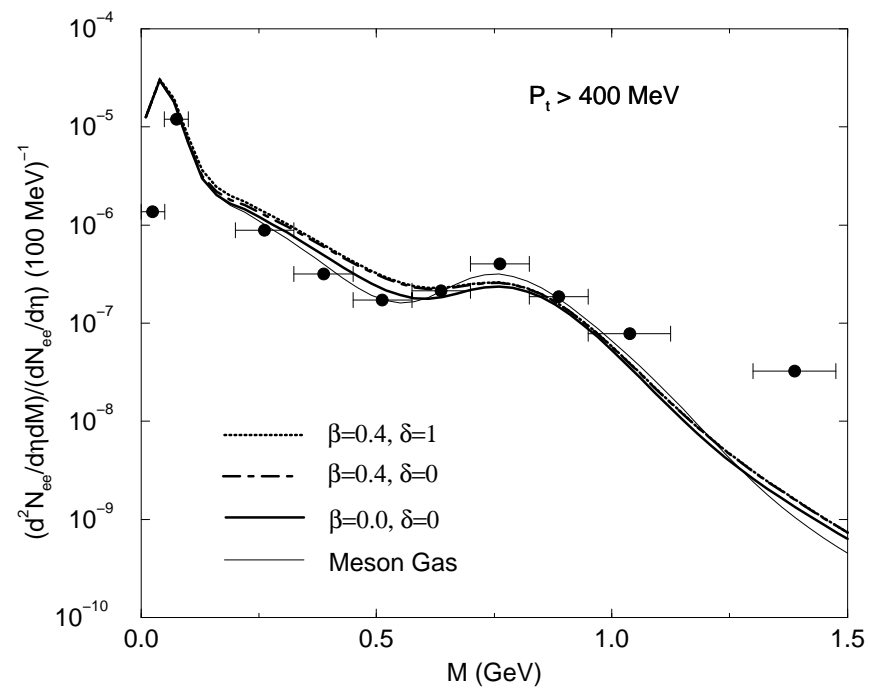

FIG. 5. Dielectron rates for high $p_{t}$ of CERES Pb-Au experiment. See text. 


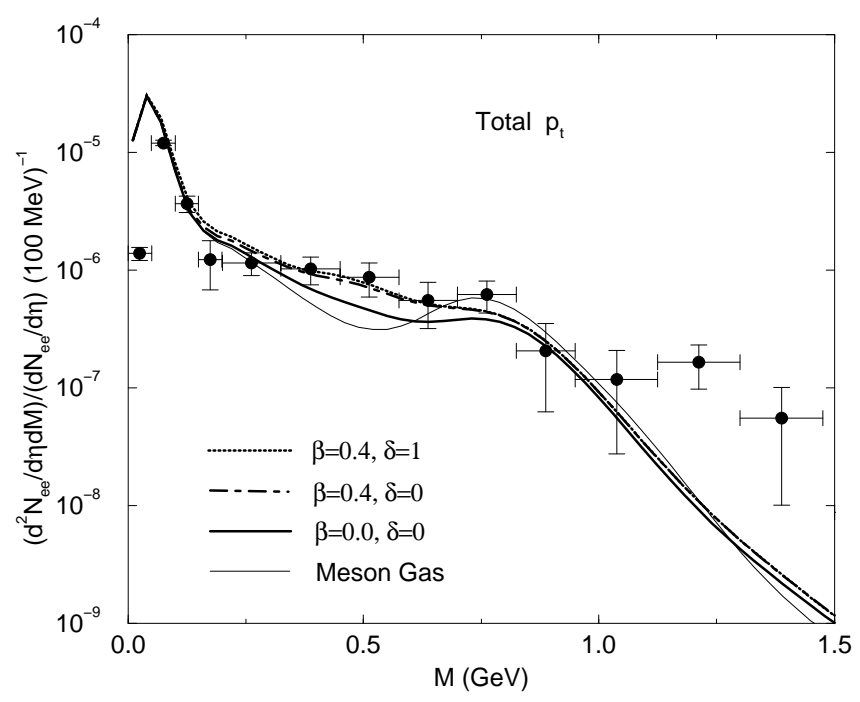

FIG. 6. Dielectron rates for the total $p_{t}$ of CERES Pb-Au experiment.

\section{ACKNOWLEDGEMENTS}

We thank a number of our colleagues at Stony-Brook for discussions. We also thank Keijo Kajantie and Su Houng Lee for their comments on the manuscript. This work was supported in part by the U.S. Department of Energy under Grant No. DE-FG02-88ER40388, and the Swedish Natural Science Research Council.

[1] M. Campostrini and A. Di Giacomo, Phys. Lett. B197 (1987) 403; S.H. Lee, Phys. Rev. D40 (1989) 2484.

[2] C. Adami, T. Hatsuda and I. Zahed, Phys. Rev. D43 (1991) 921; V. Koch and G.E. Brown, Nucl. Phys. A560 (1993) 345 ;

[3] T. Schaefer and E. Shuryak, Rev. Mod. Phys. 70 (1998) 323.

[4] R. Anishetty, J. Phys. G10 (1984) 423; K.J. Dahlem, Z. Phys. C29 (1985) 553; V.M. Belayev and V.L. Eletsky, Z. Phys. C45 (1990) 355.

[5] J. Mandula and M. Ogilvie, Phys. Lett. B201 (1988) 117.

[6] S. Nadkarni, Phys. Rev. D34 (1986) 3904.

[7] T.H. Hansson and I. Zahed, "QCD Sum Rules at High Temperature", SUNY-1990 report, unpublished; I. Zahed, "Light Relativistic Bound States in High Temperature QCD", in Thermal Field Theories, Eds. H. Ezawa et al., Elsevier 1991, p. 357; I. Zahed, "Dynamical Excitations in High Temperature QCD", in From Fundamental Fields to Nuclear Phenomena, Eds. McNeil and Price, World Scientific 1991, p. 238.

[8] K. Kajantie, M. Laine, K. Rummukainen and M. Shaposhnikov, hep-ph-9704416; and references therein.

[9] T.H. Hansson and I. Zahed, Nucl. Phys. B 292 (1987) 725.

[10] L.D. McLerran and T. Toimela, Phys. Rev. D 31, 545 (1985).

[11] H. A. Weldon, Phys. Rev. D 42, 2384 (1990).

[12] A. Patkos and N. Sakai, Nucl. Phys. B 168 (1980) 521.

[13] C.-H. Lee, H. Yamagishi, and I. Zahed, hep-ph/9806391, Phys. Rev. C, in print.

[14] G.Q. Li, C.M. Ko, and G.E. Brown, Nucl. Phys. A 606, 568 (1996).

[15] R. Rapp, G. Chanfray, J. Wambach, Nucl. Phys. A 617, 472 (1997). 